\title{
LA EVALUACION DE LA SEXUALIDAD DE LAS PERSONAS CON DISCAPACIDAD INTELECTUAL: CONSTRUCCIÓN Y DESCRIPCIÓN DE UNA ENTREVISTA SEMIESTRUCTURADA
}

\author{
Gil-Llario, M.D. \\ Dpto de Psicología Evolutiva y de la Educación \\ Universitat de València, Valencia, España \\ Dolores.Gil@uv.es \\ Ballester-Arnal, R. \\ ) Dpto de Psicología Básica, Clínica y Psicobiología \\ Universitat Jaume I, Castellón, España \\ Fernández-García, 0. \\ Dpto de Psicología Evolutiva y de la Educación \\ Universitat de València, Valencia, España \\ Morell-Mengual, $\mathbf{V}$. \\ Dpto de Psicología Evolutiva y de la Educación \\ Universitat de València, Valencia, España
}

\begin{abstract}
Recepción Artículo: 31 enero 2020 Admisión Evaluación: 4 marzo 2020 Informe Evaluador 1: 1 enero 2020

Informe Evaluador 2: 1 enero 2020 Aprobación Publicación: 20 abril 2020
\end{abstract}

Esta investigación ha sido realizada gracias a un proyecto de investigación financiado por el Programa Estatal de I+D+i Orientada a los Retos de la Sociedad del Ministerio de Economía y Competitividad (Proyecto PSI2014$53546-\mathrm{R})$.

\section{RESUMEN}

Si bien las personas con discapacidad intelectual experimentan la misma necesidad de intimidad que el resto, una serie de barreras sociales impiden la expresión de su sexualidad alentando a que se les perciba como seres asexuales. Por ello, durante mucho tiempo las voces de las personas con discapacidad no han sido escuchadas, lo que conlleva que actualmente exista una escasez de herramientas de evaluación para explorar los diferentes aspectos de la sexualidad de este colectivo teniendo en cuenta sus limitaciones en lectoescritura y comprensión. El objetivo de este estudio es la elaboración de una entrevista semiestructurada fiable y válida que contemple las características de las personas con discapacidad intelectual y posibilite la evaluación de su conducta sexual. Este instrumento, que ha sido administrado a 360 personas con discapacidad intelectual leve o moderada de entre 19 y 55 años, está formado por 34 ítems (26 de respuesta cerrada y 8 de respuesta abierta) agrupados en 4 dimensiones: comportamientos sexuales (19 ítems), conductas preventivas ( 3 ítems), información recibida sobre sexualidad (6 ítems) y experiencias de abuso sexual (6 ítems). En el proceso de construcción-validación de esta entrevista se ha visto implicado un grupo de expertos que analizaron su formato y contenido en diferentes fases. La versión final cuenta con una fiabilidad aceptable $(\boldsymbol{}=.73)$. El presente estudio ha mostrado no solo la 


\section{LA EVALUACION DE LA SEXUALIDAD DE LAS PERSONAS CON DISCAPACIDAD INTELECTUAL: CONSTRUCCIÓN Y DESCRIPCIÓN DE UNA ENTREVISTA SEMIESTRUCTURADA}

complejidad que supone elaborar una entrevista semiestructurada para explorar diferentes aspectos de la sexualidad de las personas con discapacidad intelectual, sino también la importancia de que los profesionales encargados de su aplicación cuenten con la formación necesaria.

Palabras clave: sexualidad; entrevista semiestructurada; discapacidad intelectual; construcción; descripción

\section{ABSTRACT}

The evaluation of sexuality of people with intellectual disabilities: construction and description of a semi-structured interview. Although people with intellectual disabilities experience the same need for intimacy as the rest, a series of social barriers impede the expression of their sexuality, encouraging them to be perceived as asexual beings. For this reason, for a long time the voices of people with disabilities have not been heard, which means that there is currently a shortage of assessment tools to explore the different aspects of sexuality of this group, taking into account its limitations in literacy and understanding. The objective of this study is to prepare a reliable and valid semi-structured interview that takes into account the characteristics of people with intellectual disabilities and enables the evaluation of their sexual behaviour. This instrument, which has been administered to 360 people with mild or moderate intellectual disabilities between 19 and 55 years old, is made up of 34 items ( 26 with a closed response and 8 with an open response) grouped into 4 dimensions: sexual behaviour (19 items), preventive behaviours ( 3 items), information received on sexuality (6 items) and experiences of sexual abuse (6 items). In the process of constructing-validating this interview, a group of experts was involved who analysed its format and content in different phases. The final version has an acceptable reliability $(\boldsymbol{V}=.73)$. This study has shown not only the complexity of preparing a semi-structured interview to explore different aspects of the sexuality of people with intellectual disabilities, but also the importance of the professionals in charge of its application having the necessary training.

Keywords: sexuality; semi-structured interview; intellectual disability; building; description

\section{INTRODUCCIÓN}

La sexualidad, como componente multidimensional del ser humano que comprehende las emociones, la intimidad, el erotismo, el placer y las relaciones con otros, está presente durante todo el ciclo vital. A pesar de ello, las personas con discapacidad intelectual (DI) constituyen un subgrupo al que tradicionalmente se le ha negado la expresión de su sexualidad dada la influencia de mitos, estereotipos y falsas creencias que han fomentado actitudes negativas hacia este grupo y la consecuente elusión de su derecho a satisfacer sus necesidades sexuales y emocionales (Franco, Cardoso y Neto 2012; Olavarrieta et al.2013). Parte de la sociedad considera que este colectivo no puede tomar decisiones apropiadas y presenta comportamientos sexuales peligrosos y agresivos por la falta de control de sus impulsos, creencias que hasta el momento no han recibido sustento científico. Así, aunque los aspectos relacionados con la salud sexual de este subgrupo despiertan cada vez más interés (Parchomiuk, 2012), todavía existe una falta sustancial de conocimiento sobre el desarrollo sexual de las personas con DI y los desafíos que enfrentan (Kijak, 2013).

Investigaciones recientes muestran que alrededor del $85 \%$ de las personas con DI dice haber realizado prácticas sexuales con o sin penetración con otra persona (Bernert y Ogletree, 2013), aunque el autoerotismo se postula como el comportamiento sexual comúnmente practicado (Díaz, Gil, Ballester, Morell y Molero, 2014; Gil Llario, Morell Mengual, Ballester Arnal y Díaz Rodríguez, 2018), sobre todo entre los hombres (Kijak, 2013). Además, aunque la mayoría asegura haber recibido información sobre el uso de anticonceptivos (Gil Llario, Morell Mengual, Ballester Arnal y Díaz Rodríguez, 2018), dicha información no se ajusta a sus capacidades y son los padres y profesionales quienes acaban decidiendo cuál es el método anticonceptivo más pertinente (Olavarrieta et al.2013). Así mismo, se considera que las personas con DI son víctimas de abuso sexual en mayor proporción que la población general, aunque se carece de cifras válidas acerca de la prevalencia real del abuso sexual en este colectivo (GilLlario, Ballester-Arnal, Fernández-García, Caballero-Gascón y Morell-Mengual, 2018). 
A todo lo expuesto cabe añadir que las personas con DI presentan niveles más bajos de conocimientos sobre sexualidad que la población general y las personas con discapacidad física (Borawska-Charko, Rohleder y Finlay, 2016; Jahoda y Pownall, 2014), aunque la mayoría de los adultos que integran este grupo expresan su deseo de hablar más sobre sexualidad (Karellou, 2017). Además, cuando se ha proporcionado educación sexual ha sido inadecuada e insuficiente, ya que no se han tenido en cuenta sus limitaciones en el aprendizaje. Con ello, se evidencia la necesidad de realizar un cambio en la metodología de evaluación e intervención de forma que pueda garantizarse la expresión adecuada de las inquietudes que ocupan a este colectivo en un clima abierto, respetuoso y positivo (McDaniels y Fleming, 2016).

Comprender cómo se desarrolla y funciona la sexualidad de este grupo social es esencial para elaborar e implementar programas de educación afectivo-sexual efectivos (Gil Llario, Morell Mengual, Ballester Arnal y Díaz Rodríguez, 2018). Pero, a la hora de extraer información a este colectivo nos enfrentamos a sus limitaciones intelectuales, así como en habilidades comunicativas y sociales, lo cual dificulta mucho el proceso de evaluación. Por ello, en muchos casos se recurre a los familiares y profesionales como informadores directos, quienes frecuentemente desatienden el discurso de la propia víctima obteniéndose información parcial (González y Ramos, 2016).

Con el objetivo de dar voz a este colectivo y paliar esta falta de instrumentos de evaluación efectivos que posibiliten la expresión de la sexualidad de las personas con DI (Kramers-Olen, 2017), se desarrolla el presente estudio dirigido a elaborar y describir una entrevista semiestructurada que permita recoger información sobre su conducta sexual desde un enfoque que contemple las limitaciones en la comunicación expresiva y receptiva de las personas con DI y su diversidad sexual.

\section{MÉTODO}

\section{Participantes}

La muestra está compuesta por 360 personas adultas con diferente grado de DI que son beneficiarias de algún tipo de recurso asistencial, bien un centro ocupacional bien un servicio de estancia diurna. Estos centros están ubicados en Castilla La Mancha, en las provincias de Albacete, Cuenca y Ciudad Real, y en Andalucía, en las provincias de Cádiz y Granada. Un tercio de los usuarios acude a estos recursos procedente de sus domicilios familiares donde residen, otro tercio llega desde viviendas tuteladas, y el otro tercio vive en centros residenciales y realiza los diferentes talleres y actividades en el propio recurso.

Tabla 1. Características sociodemográficas de las personas con DI

\begin{tabular}{lc}
\hline & $\mathbf{N}(\%)$ \\
\hline Edad & \\
$\quad$ Media (DT) & $\begin{array}{c}(19-57) \\
(\mathrm{DT}=10.50)\end{array}$ \\
Rango & $180(50 \%)$ \\
\hline Género & $180(50 \%)$ \\
$\quad$ Hombre & $346(96.1 \%)$ \\
$\quad$ Mujer & $14(3.9 \%)$ \\
\hline Orientación sexual & \\
$\quad$ Heterosexual & \\
$\quad$ Homosexual & $212(58.9 \%)$ \\
\hline Grado de & $148(41.1 \%)$ \\
discapacidad & \\
$\quad$ Leve & $120(33.3 \%)$ \\
$\quad$ Moderada & $120(33.3 \%)$ \\
Lugar de residencia & $120(33.3 \%)$ \\
Centro &
\end{tabular}

International Journal of Developmental and Educational Psychology 


\section{LA EVALUACION DE LA SEXUALIIDAD DE LAS PERSONAS CON DISCAPACIDAD INTELECTUAL: CONSTRUCCIÓN Y DESCRIPCIÓN DE UNA ENTREVISTA SEMIESTRUCTURADA}

La mitad de los participantes son mujeres y la otra mitad hombres. Respecto a la edad, casi la mitad de la muestra tiene entre 45 y 55 años (45.8\%), mientras que el 28.3\% tiene entre 31 y 44 años y el 25.8\% entre 18 y 30 años. Su cociente intelectual (Cl) oscila entre 50 y 85, presentando la mayoría (58.9\%) una discapacidad intelectual leve ( $\mathrm{Cl}$ entre 50 y 70 ) y el $41.1 \%$ restante una discapacidad intelectual moderada ( $\mathrm{Cl}$ entre 71 y 85 ) (Ver Tabla 1). En relación a la orientación sexual, la mayor parte de los participantes eran heterosexuales (96.1\%), mientras el 3.9\% se identifica como homosexual. En el momento que se realizó la investigación el $84.2 \%$ de los participantes tenía pareja.

Todos los participantes fueron reclutados mediante muestreo incidental no aleatorio, tratando de compensar diversas características sociodemográficas como la edad, el género y el lugar de residencia. Los criterios de inclusión fueron poseer certificado de discapacidad intelectual leve o moderada, contar con suficientes competencias verbales para poder responder los cuestionarios y tener capacidad para dar su consentimiento libremente.

\section{Instrumento}

Cuestionario Ad-Hoc de Información Sociodemográfica. Se trata de un cuestionario que recoge información sobre el género (mujer y hombre), la edad, el grado de discapacidad (leve o moderada) y el lugar de residencia (vivienda tutelada, centro residencial y unidad familiar).

CUSEXDI. Cuestionario sobre sexualidad para personas con Discapacidad Intelectual. Este instrumento fue elaborado ad hoc por Gil-Llario y Díaz-Rodríguez (2013) para la recogida de datos en el marco de un proyecto de investigación sobre la salud sexual de las personas adultas con DI. Se trata de una entrevista semiestructurada conformada por 34 ítems, 26 de respuesta cerrada y 8 de respuesta abierta, que evalúan diversos aspectos de la sexualidad de las personas con DI (comportamientos sexuales, conductas preventivas, información recibida sobre sexualidad y experiencias de abuso sexual). Puede ser auto o heteroaplicada, dependiendo de las limitaciones en lectoescritura y comprensión que presente el sujeto evaluado. Su ámbito de aplicación es el de las personas con DI y se cumplimenta en unos 20 minutos aproximadamente según las características del participante.

\section{Procedimiento}

A continuación, se describe el proceso de construcción del CUSEXDI como instrumento de recogida de información sobre la sexualidad de las personas con DI.

En una fase previa al diseño del instrumento, profesionales expertos en investigación, salud sexual y DI, realizaron una exhaustiva revisión bibliográfica sobre las investigaciones e instrumentos de evaluación publicados hasta el momento, prestando especial atención a las áreas que se evaluaban. A pesar de la escasez de estudios que exploran la conducta sexual de las personas con DI, la información encontrada permitió concretar el contenido y características estructurales que debía tener el instrumento dado el perfil de los participantes. Posteriormente, las ideas recogidas por el grupo de expertos se pusieron en común con algunos profesionales que trabajaban con personas con DI. Estos últimos aportaron una visión verdaderamente importante al tener en cuenta la realidad a la que se enfrentan diariamente en los centros residenciales y ocupacionales. Por ejemplo, reafirmaron la importancia de indagar sobre posibles experiencias de abuso sexual dada la frecuencia de casos, y propusieron incluir preguntas más detalladas sobre sus relaciones de pareja, ya que comúnmente confunden los diferentes tipos de relaciones (relaciones de pareja, de amistad, etc.).

Una vez concretados los aspectos de la sexualidad de las personas con DI sobre los que se quería recoger información se procedió a redactar los reactivos. Se tuvo en cuenta la inclusión de ítems tanto de respuesta abierta que permiten crear un ambiente más distendido y recabar información más detallada, como de respuesta cerrada, de opción única y de opción múltiple, que permiten obtener información más precisa y clara en menor tiempo. Esto nos permite recoger información objetiva sobre ciertos comportamientos sexuales experimentados por los participantes, pero también más subjetiva sobre sentimientos y emociones surgidos. Posteriormente, se proporcionó al instrumento un formato de entrevista, ya que dadas las características de la población diana y el contenido de carácter personal e íntimo sobre el que se pregunta, se creyó conveniente elaborar un instrumento cua- 
litativo que permitiera establecer mayor rapport con la persona evaluada creando un ambiente de total confianza. Así mismo, teniendo en cuenta la percepción del grupo de profesionales que trabajan con personas con DI en los recursos, se le otorgó al instrumento un formato que permitiera su administración también de forma autoaplicada para proporcionarle esta alternativa a los sujetos que, por el contenido de la entrevista, prefieran contestarlo de forma más íntima y presenten un buen nivel en lectoescritura y la suficiente comprensión lectora como para hacerlo. La escala quedó conformada por 34 ítems distribuidos en 4 dimensiones de acuerdo a su contenido: comportamientos sexuales, conductas preventivas, información recibida sobre sexualidad y experiencias de abuso sexual.

En una fase posterior, se presentó la última versión de la entrevista semiestructurada a los profesionales de los centros residenciales y ocupacionales encargados de la evaluación de las personas con DI que participaban en el estudio, y se les capacitó para su implementación. Realizar esta formación fue realmente importante ya que permitió establecer unos criterios estándar para realizar el pase de la entrevista, evitando, en la medida de lo posible, la influencia del evaluador en las respuestas de los participantes, sobre todo en aquellas preguntas que son abiertas. Posteriormente, los profesionales encargados de la recogida de datos realizaron un pase piloto con una muestra pequeña de sujetos.

Finalmente, se aplicó la entrevista a la muestra seleccionada que cumplía los criterios de inclusión y se procedió al registro y codificación de los datos usando el paquete estadístico SPSS.

\section{Análisis de datos}

En el análisis descriptivo del grupo muestral y de los ítems se emplearon estadísticos descriptivos. Para informar sobre la fiabilidad del instrumento se calculó el alfa de Cronbach. Todos los análisis estadísticos se realizaron con el programa IBM SPSS Statistics 23.

\section{RESULTADOS}

\section{Descripción del contenido}

El Cuestionario sobre sexualidad para personas con Discapacidad Intelectual consta de varias dimensiones que le dan sentido a la composición del instrumento:

Comportamiento sexual: En esta dimensión se incluyen 19 ítems que hacen referencia a la expresión conductual de la sexualidad personal ligada a la obtención de placer. Con esta dimensión pretendemos obtener información sobre los cambios físicos percibidos en la pubertad, el interés y atracción sexual hacia miembros de otro sexo 0 del mismo, las relaciones de pareja disfrutadas, las prácticas sexuales realizadas, así como otras vivencias relacionadas con la sexualidad como las fantasías sexuales. También se incluyen algunos ítems que indagan sobre la forma de vivenciar y sentirse frente a ciertos comportamientos sexuales.

Conducta preventiva: Constituida por 3 ítems se convierte en la dimensión con menos número de reactivos. En ella se incluyen aquellos ítems relacionados con el conocimiento y uso de los diferentes métodos anticonceptivos, indagando sobre si han recibido información sobre ello previamente y quién se la proporcionó.

Información recibida sobre sexualidad: Los 6 ítems que forman parte de esta dimensión indagan sobre la formación e información sobre sexualidad que ha recibido previamente el evaluado. Hace hincapié en el interés del participante en recibir este tipo de información, la edad en la que le hablaron sobre esto, quién lo hizo y cómo se sintió, ya que esto último influirá notablemente en su actitud hacia la sexualidad. Además, se intenta detallar qué aspectos de la sexualidad tienen mayor relevancia para el evaluado.

Experiencias de abuso sexual: Se trata de una dimensión conformada por 6 ítems que indagan sobre posibles experiencias de abuso sexual. Pero, los reactivos incluidos no tienen como objetivo único la detección de casos de violencia sexual, sino también preguntan sobre detalles que permiten garantizar la calidad de la revelación evitando falsos positivos. Al mismo tiempo también se indaga sobre las consecuencias personales derivadas de la experiencia. 


\section{LA EVALUACION DE LA SEXUALIDAD DE LAS PERSONAS CON DISCAPACIDAD INTELECTUAL: CONSTRUCCIÓN Y DESCRIPCIÓN DE UNA ENTREVISTA SEMIESTRUCTURADA}

\section{Propiedades de los ítems}

La tabla 2 incluye la relación de ítems que conforman el CUSEXDI y la distribución de las respuestas de los adultos evaluados entre las diferentes alternativas. En este caso, esto nos permite obtener una visión global del funcionamiento de los ítems y de las alternativas de respuesta.

Por lo que respecta a los ítems, se considera que todos cumplen con su propósito, ya que abordan aspectos diferentes y complementarios sin redundar. De los 8 reactivos de respuesta abierta, en dos de ellos (ítems 9 y 31 ) se tomó la decisión de incluir alternativas dicotómicas (Sí/No) dando la opción de ampliar información mediante el relato de los evaluados. Así mismo, en el ítem 8 se decidió fusionar las alternativas "No" y "No me acuerdo" a la hora de realizar la codificación ya que, aunque presentan matices diferentes, se solapaban las respuestas de los participantes. En el ítem 14, también se fusionaron las alternativas "besos" y "caricias", ya que se entendió que eran prácticas sexuales que requieren un nivel de intimidad semejante y los participantes reaccionaban de igual forma a ambas alternativas. Además, se eliminó la alternativa "Otro" por la ausencia de respuestas, al igual que sucedió en el ítem 18. Este también fue el caso de la alternativa "No lo sé" del ítem 17. Respecto a los ítems 26 y 27 se decidió incluir la alternativa de respuesta "No contesta", ya que por el carácter invasivo del contenido de las preguntas muchos participantes no querían contestar y cabe respetar su decisión.

Tabla 2. Ítems del cuestionario CUSEXDI y distribución de las respuestas de los participantes.

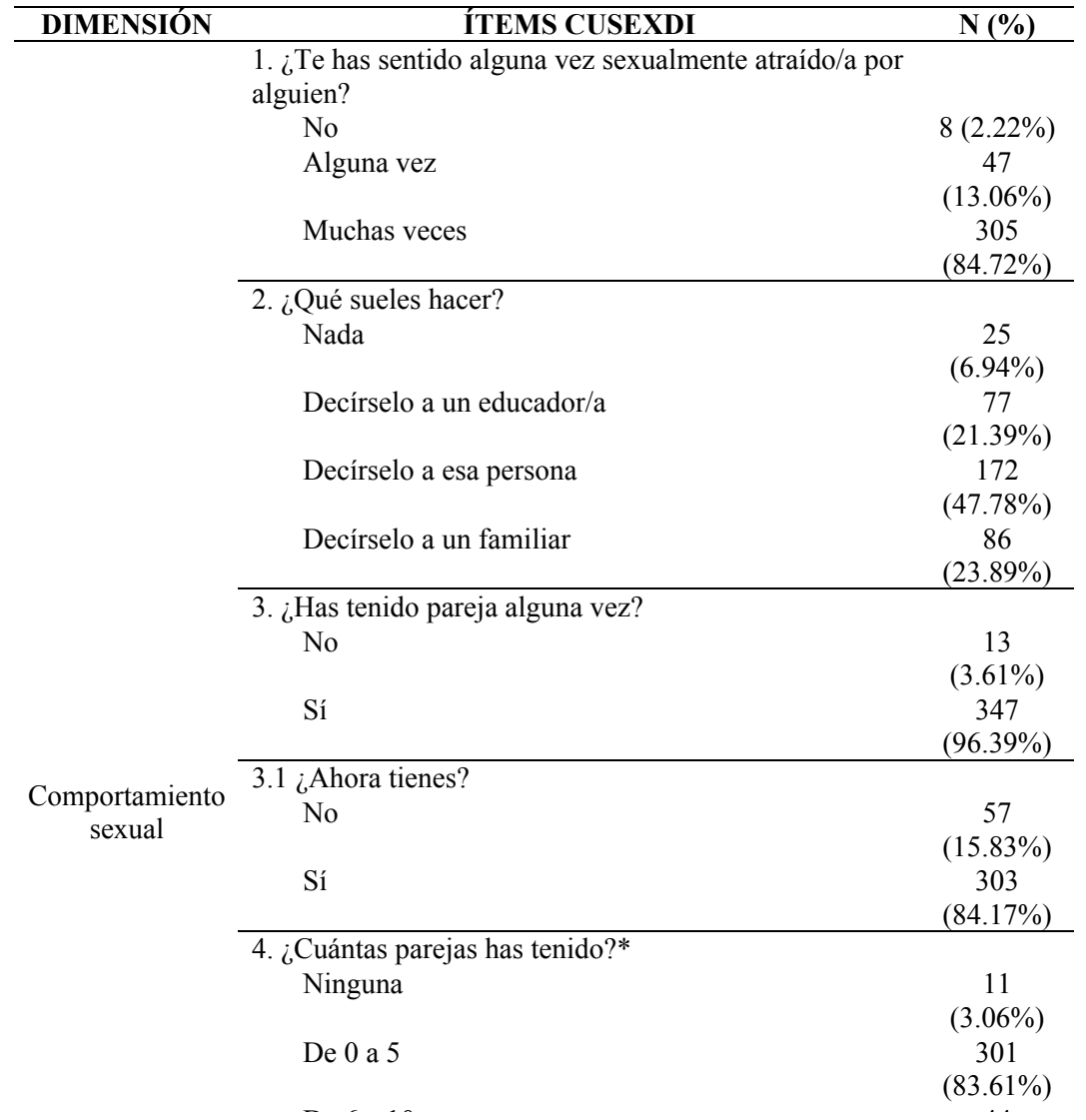


De 6 a 10

Más de 10

5. ¿Cuánto tiempo has durado como máximo?* Menos de 1 año

Entre 1 y 2 años

Más de 2 años

6. ¿Te gusta alguien ahora? No

$(87.78 \%)$

7. ¿Te suelen gustar personas del otro sexo o del tuyo?

Del otro sexo

De mí mismo sexo

8. ¿Has tenido despierto/a o dormido/a fantasías o sueños sexuales?

No/No me acuerdo

Algunas veces

Muchas veces

9. ¿Puedes contar una fantasía o un sueño que hayas tenido hace poco?*

No

Sí $72(20 \%)$

10. ¿Te has masturbado alguna vez?

No $\quad 36(10 \%)$

$\frac{\text { Sí }}{11 . \text { ¿A qué edad empezaste?* }}$

Nunca

$324(90 \%)$

Entre $\operatorname{los} 7$ y 12 años

Entre los 13 y 16 años

Entre los 17 y 20 años

12. ¿Con qué frecuencia lo haces?

Nunca

$27(7.5 \%)$

Algunas veces al año (3 o 4)

$7(1.94 \%)$

Algunas veces al mes ( 1 o 2$)$

Algunas veces a la semana ( 1 o 2$)$ 
13. ¿Has tenido alguna vez relaciones sexuales? No

14. ¿De qué tipo? (se puede señalar más de una)

Besos y caricias

Sexo oral

Sexo vaginal

Sexo anal

29. ¿Tus padres te dejarían tener novio/a?

No

29.1 ¿Por qué?

Porque está mal

Porque yo no puedo hacer esas cosas

Claro que sí, como todo el mundo

30. ¿Te gustaría tener novio/a?

Nunca

Más adelante quizá

$\mathrm{Si}$

32. Edad primera menstruación*

Entre los 10 y 12 años

Entre los 13 y 15 años

Entre los 16 y 18 años

33. Edad inicio primeros síntomas de pubertad*

Entre los 10 y 12 años

Entre los 13 y 15 años

Entre los 16 y 18 años

34. ¿Cuál es tu nivel de satisfacción sexual?

Nada satisfactorio

Poco satisfactorio 


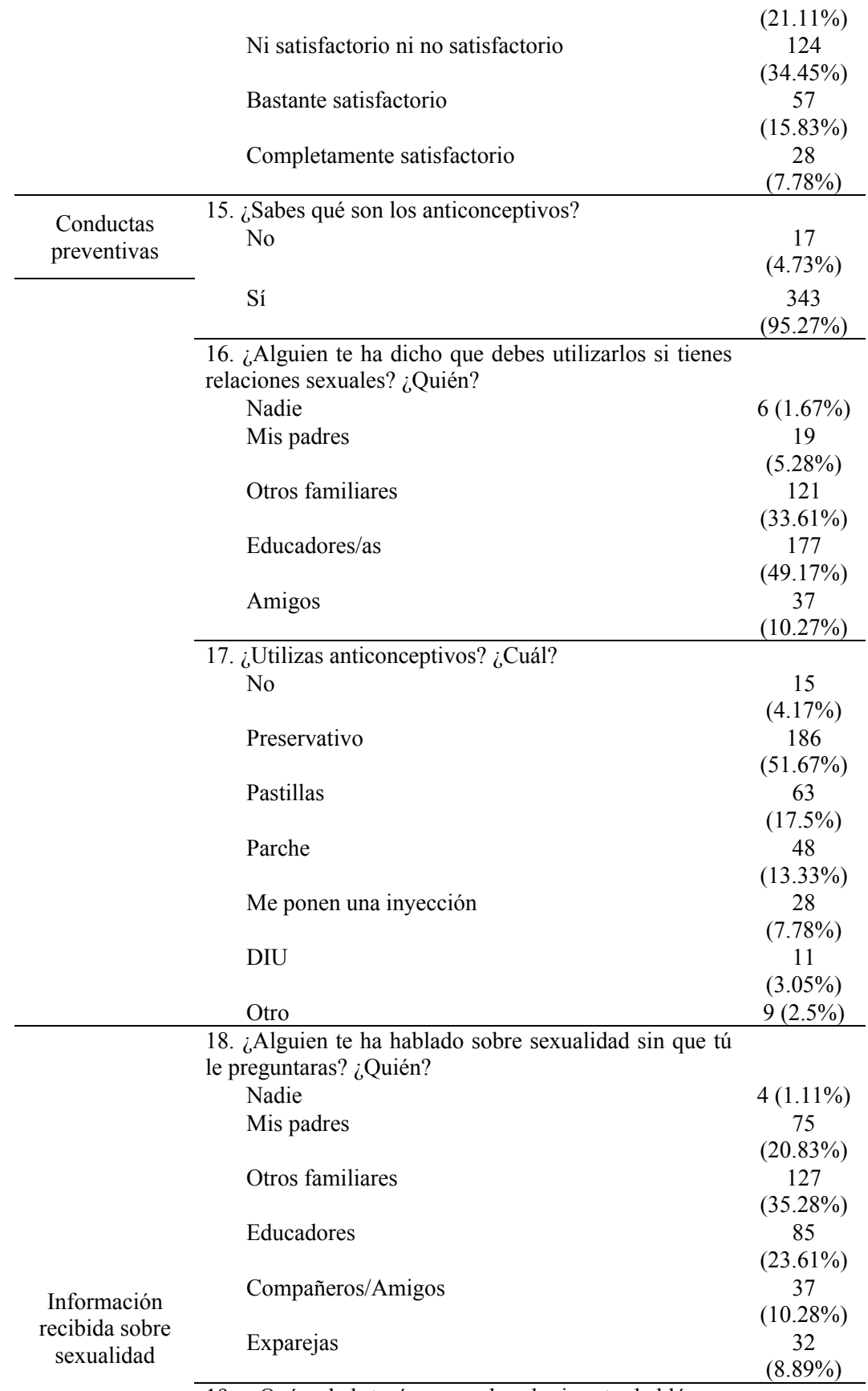

19. ¿Qué edad tenías cuando alguien te habló por primera vez de sexualidad?* 
Entre los 10 y 13 años

Entre los 14 y 17 años

Entre los 18 y 20 años

20. ¿Le has preguntado a alguien sobre sexualidad? ¿A quién?

A nadie

A mis padres

A otros familiares

A educadores/as

A compañeros/amigos

\section{1. ¿Cómo te sentiste?}

Bien, volveré a preguntar

Me dio mucha vergüenza preguntar pero volvería a $108(30 \%)$ hacerlo

No volveré a preguntar cosas sucias

22. ¿Te gustaría hablar más sobre sexualidad?

No

Sí

$(88.61 \%)$

31. ¿Tienes alguna pregunta que quieras hacer sobre sexualidad?*

No

Sí

23. ¿Alguna vez alguien ha intentado tener relaciones sexuales contigo a pesar de que tú no quisieras?

No

Sí

24. ¿Te hizo daño?

No

Sí

Experiencias de abuso sexual

25. ¿Se lo dijiste a alguien?

No

$(5.28 \%)$

Sí

26. ¿A quién? 
No contesta

A mi madre

$1(0.28 \%)$

A mi padre

$1(0.28 \%)$

A otro familiar

$3(0.83 \%)$

A un educador

$5(1.39 \%)$

A un amigo

$1(0.28 \%)$

27. ¿Cómo reaccionó?

No contesta

No se lo creyó

Me riño y me dijo que yo tenía la culpa

Me dijo que no me preocupara y lo arregló todo

$4(1.11 \%)$

28. ¿Desde entonces has cambiado?

No

Sí

28.1. ¿Sientes miedo, enfado, etc. cuando sale el tema del sexo?

No / No contesta

Siento miedo

Siento enfado

*Ítems de respuesta abierta (se muestra la codificación que se ha realizado con posterioridad de acuerdo a las respuestas de los participantes).

\section{Consistencia interna}

La consistencia ha sido extraída mediante el coeficiente Alfa de Cronbach para la entrevista global obteniéndose un valor de .73 .

\section{Viabilidad}

En cuanto a la viabilidad, observamos que el CUSEXDI fue valorado positivamente por parte de los profesionales expertos, quienes consideraron que tanto los reactivos como las dimensiones incluidas en el instrumento eran necesarias, útiles y se ajustaban al perfil del grupo social al que va dirigido, ya que no quedó ningún ítem por responder en ninguna entrevista y el tiempo medio de administración fue adecuado (20 minutos aproximadamente).

\section{CONCLUSIONES}

El objetivo del presente estudio ha sido desarrollar un instrumento fiable y válido que permita evaluar la conducta sexual de las personas con DI, teniendo en cuenta las limitaciones y características de dicho colectivo. Tras el análisis de la entrevista semiestructurada creada (CUSEXDI) podemos concluir que este instrumento no solo puede ser útil a la hora de obtener información sobre los comportamientos sexuales de las personas con DI, sino también para comprobar en qué medida estas personas son capaces de traducir y poner en práctica los conocimientos recibidos sobre educación sexual (Schaafsma, Kok, Stoffelen y Curfs, 2017). Se trata de un aspecto de especial relevancia ya que esto puede actuar como indicador de eficacia de los programas de educación afectivo-sexual, determinando no solo si son efectivos para ese grupo social sino también hacia dónde deben orientarse intervenciones futuras de carácter primario o secundario (Recio et. al., 2014).

Por lo que se refiere a las características psicométricas del instrumento, los ítems presentan una buena con- 


\section{LA EVALUACION DE LA SEXUALIDAD DE LAS PERSONAS CON DISCAPACIDAD INTELECTUAL: CONSTRUCCIÓN Y DESCRIPCIÓN DE UNA ENTREVISTA SEMIESTRUCTURADA}

sistencia interna, mostrando una fiabilidad aceptable. Así mismo, cabe resaltar el método de evaluación que emplea el Cuestionario sobre sexualidad para personas con DI al usar un formato de entrevista semiestructurada. Como destaca Kramers-Olen (2017) las evaluaciones de puntaje único no deben reemplazar otras metodologías cualitativas como las entrevistas semiestructuradas, ya que estas últimas pueden ser más beneficiosas a la hora de identificar fortalezas y debilidades, informar sobre objetivos educativos y/o terapéuticos y reforzar los derechos de las personas con DI en la expresión sexual.

En otro orden de cosas, este proceso de elaboración y descripción nos ha permitido reflexionar alrededor de la importancia de valorar todos los factores que influyen en una evaluación, y en especial sobre el papel del evaluador durante la administración de la entrevista dado que se convierte en un elemento imprescindible. Así, sería aconsejable que el profesional actuara de mediador entre el instrumento y el evaluado, proporcionando una explicación adaptada del mismo que asegure su comprensión. Esto contribuiría a una mejor comprensión de los conceptos al proporcionar una explicación más rica y personalizada. Por ello, se hace evidente, que el profesional que realice las evaluaciones debe poseer amplios conocimientos no solo sobre el instrumento en sí (qué evalúa cada ítem, cómo es su formato de respuesta, etc.), sino también sobre los usuarios a los que se les va a pasar (nivel de comprensión, nivel en lecto-escritura, etc.) (Gil-Llario et al., 2018).

A modo de conclusión cabe añadir que cuando se reconocen los derechos sexuales y la capacidad de elección de las personas con discapacidad, se transmite una sensación de respeto mutuo que refuerza el bienestar emocional de todos los miembros de la sociedad, de ahí la importancia de dar voz a este colectivo.

\section{REFERENCIAS BIBLIOGRÁFICAS}

Bernert D.J. y Ogletree R.J. (2013) Women with intellectual disabilities talk about their perceptions of sex. Journal of Intellectual Disability Research, 57, 240-9. doi:10.1111/j.1365-2788.2011.01529.x.

Borawska-Charko, M., Rohleder, P. y Finlay, W.M. (2016). The Sexual Health Knowledge of People with Intellectual Disabilities: a Review. Sexuality Research and Social Policy, 14, 393-409.

Díaz I.M., Gil M.D., Ballester R., Morell V.J. y Molero R.J. (2014) Knowledge, attitudes and sexual behavior in adults with intellectual disabilities. International Journal of Developmental and Educational Psychology, 1, 415-22. doi: 10.17060/ijodaep.2014.n1.v3.519.

Franco, D. G., Cardoso, J. y Neto, I. (2012) Attitudes towards affectivity and sexuality of people with intellectual disability. Sexuality and Disability, 30, 261-87. doi:10.1007/s11195-012-9260-x.

Friedman, C., Arnold, C.K., Owen, A.L., y Sandman, L. (2014). 'Remember our voices are our tools': Sexual selfadvocacy as defined by people with intellectual and developmental disabilities. Sexuality and Disability, 32, $515-532$.

Gil-Llario, M.D., Ballester-Arnal, R., Fernández-García, 0., Caballero-Gascón, L. y Morell-Mengual, V. (2018). La evaluación del abuso sexual en personas con discapacidad intelectual: proceso de construcción y validación de un instrumento de autoinforme. International Journal of Developmental and Educational Psychology, 1(2), 111-118.

Gil-Llario, M.D. y Díaz-Rodríguez, I. (2013). Análisis del comportamiento sexual, así como de las necesidades y los deseos sexuales de un colectivo de discapacitados intelectuales adultos (tesis de postgrado). Universitat de València, Valencia.

Gil Llario, M.D., Morell Mengual, V., Ballester Arnal, R., y Díaz Rodríguez, I. (2018). The experience of sexuality in adults with intellectual disability. Journal of Intellectual Disability Research, 62(1), 72-80. doi: 10.1111/jir.12455

Karellou, I. (2017) "It Is Only Natural....": Attitudes of Young People With Intellectual Disabilities Toward Sexuality in Greece. Journal of Mental Health Research in Intellectual Disabilities, 1033), 217-236, doi: 10.1080/19315864.2016.1278291

Jahoda, A., y Pownall, J. (2014). Sexual understanding, sources of information and social networks; the reports 
of young people with intellectual disabilities and their non-disabled peers. Journal of Intellectual Disability Research, 58, 430-441. doi:10.1111/jir.12040

Kijak R. (2013) The sexuality of adults with intellectual disability in poland. Sexuality and Disability, 31, 109-23. doi: 10.1007/s11195-013-9294-8.

Kramers-Olen, A. (2017). Quantitative assessment of sexual knowledge and consent capacity in people with mild to moderate intellectual disability. South African Journal of Psychology, 47(3), 367-378.

McDaniels, B. y Fleming, A. (2016) Sexuality education and intellectual disability: time to address the challenge. Sexuality and Disability, 34, 215-25. doi: 10.1007/s11195-016-9427-y.

Olavarrieta, S., Darín, L., Suárez, P., Tur, N., Besteiro, B. y Gómez-Jarabo, G. (2013) Attitudes towards sexuality, sterilization, parenting and nurture skills of people with intellectual disability: a preliminary study. Siglo Cero, $44,55-69$.

Parchomiuk M. (2012) Specialists and sexuality of individuals with disability. Sexuality and Disability, 30, 40719. doi: 10.1007/s11195-011-9249-x.

Recio, M., Galindo, L., Cendra, J., Alemany, A., Martorell, A, y Villaró, A. (2014) Abuso y discapacidad intelectual: Orientaciones para la prevención y la actuación. FEAPS Madrid y Fundación Carmen Pardo-Valcarce

Schaafsma, D., Kok, G., Stoffelen, J.M.T., y Curfs, L.M.G. (2017). People with intellectual disabilities talk about sexuality: Implications for the development of sex education. Sexuality and Disability, 35, 21-38. 
